\title{
Bacterial Contamination and Disinfection Status of Laryngoscopes Stored in Emergency Crash Carts
}

\author{
Jae Hyung Choi', Young Soon Cho' ${ }^{2}$ Jung Won Lee', Hee Bong Shin ${ }^{3}$, In Kyung Lee \\ ${ }^{1}$ Department of Emergency Medicine, Soonchunhyang University College of Medicine, Cheonan; ${ }^{2}$ Department of Emergency Medicine, Soonchun- \\ hyang University College of Medicine, Bucheon; ${ }^{3}$ Department of Laboratory Medicine, Soonchunhyang University College of Medicine, Bucheon; \\ ${ }^{4}$ Department of Nursing, Soonchunhyang University College of Medicine, Bucheon, Korea
}

Objectives: To identify bacterial contamination rates of laryngoscope blades and handles stored in emergency crash carts by hospital and area according to the frequency of intubation attempts.

Methods: One hundred forty-eight handles and 71 blades deemed ready for patient use from two tertiary hospitals were sampled with sterile swabs using a standardized rolling technique. Samples were considered negative (not contaminated) if no colonies were present on the blood agar plate after an 18-hour incubation period. Samples were stratified by hospital and according to the frequency of intubation attempts (10 attempts per year) using the $\chi^{2}$-test and Fisher exact test.

Results: One or more species of bacteria were isolated from 4 (5.6\%) handle tops, 20 (28.2\%) handles with knurled surfaces, and 27 (18.2\%) blades. No significant differences were found in microbial contamination levels on the handle tops and blades between the two hospitals and two areas according to the frequency of intubation attempts. However, significant differences were found between the two hospitals and two areas in the level of microbial contamination on the handles with knurled surfaces $(p<0.05)$.

Conclusions: Protocols and policies must be reviewed to standardize procedures to clean and disinfect laryngoscope blades and handles; handles should be re-designed to eliminate points of contact with the blade; and single-use, one-piece laryngoscopes should be introduced.

Key words: Laryngoscopes, Equipment contamination, Disinfection, Hospitals, South Korea

\section{INTRODUCTION}

A laryngoscope equipped within emergency crash carts at a hospital is regarded as essential for emergency airway management; additionally, it is indicated as the main reason for

Received: January 31, 2017 Accepted: March 14, 2017

Corresponding author: Young Soon Cho, MD

170 Jomaru-ro, Wonmi-gu, Bucheon 14584, Korea

Tel: +82-32-621-5119, Fax: +82-32-621-6950

E-mail: emer0717@gmail.com

This is an Open Access article distributed under the terms of the Creative Commons Attribution Non-Commercial License (http://creativecommons.org/licenses/by$\mathrm{nc} / 4.0 /$ ) which permits unrestricted non-commercial use, distribution, and reproduction in any medium, provided the original work is properly cited. nosocomial infection, as it is an invasive instrument that directly contacts a patient's mucous membrane, saliva, and blood during endotracheal intubation [1,2]. In South Korea (hereafter Korea), there are differences among each report of inpatients' nosocomial infection rate (3-15\%) [3,4]; moreover, an immunocompromised patient or infant's nosocomial infection is the main reason for morbidity and mortality. Thus, infection management of invasive medical instruments such as laryngoscopes is considered mandatory $[5,6]$. According to disinfection instructions for instruments and goods used by medical institutions provided by the Ministry of Health and Welfare and US Centers for Disease Control and Prevention (CDC), the laryngoscope blade is classified as a semi-critical instrument, suggest- 
ing that it requires high-level disinfection or sterilization [7-9]. A semi-critical instrument is an instrument that contacts the mucous membrane or damaged skin and requires high-level disinfection to destroy all types of microorganisms, excluding bacterial spores; examples include respiratory therapy and anesthesia equipment, some endoscopes, laryngoscope blades, and esophageal manometry probes.

The handle of a laryngoscope is classified as a non-critical instrument, suggesting that it requires intermediate-level disinfection [7-9]. A non-critical instrument is an instrument that contacts the skin without damaging it, but it does not make contact with a mucous membrane; examples include bedpans, blood pressure cuffs, crutches, computers, and stethoscopes. Usually these instruments require low-level disinfection to eliminate bacteria, viruses, and some of fungi, not mycobacteria or bacterial spores. However, intermediate-level disinfection is required for blood-stained non-critical instruments to eliminate bacteria, viruses, yeasts, and mycobacteria, except bacterial spores. However, there is no standardized guideline for infection management of laryngoscopes to date, and each manufacturer of laryngoscopes and even hospitals suggest different disinfection methods $[3,10,11]$. The laryngoscope handle is a possible source of infection $[12,13]$, with a direct route through the handle head's top and indirect route through the handle's knurled surface. Part of the handle head's top connects to the blade and handle, and sometimes it makes contact with the oral cavity during the intubation process, resulting in a direct infection route. The laryngoscope handle's knurled surface is processed as a grid-type knurl to increase its frictional force, making it difficult to clean, which increases the residual rate of blood or bacteria [12-14]. The handle's knurled surface touches the blade when the laryngoscope blade is folded, causing contamination or contaminating medical team members' hands, which is regarded as an indirect infection route. The laryngoscope blade is frequently folded before intubation to check that it is functioning normally and to reduce its battery consumption (Figure 1).

Two target hospitals and their emergency crash carts each included more than two laryngoscope blades and handles that were managed by nurses based on the same disinfection management instructions. The laryngoscope blade is disinfected using peracetic acid (Antec International Ltd., Suffolk, UK) or ortho-phthalaldehyde (Ethicon Inc., Irvine, CA, USA) from sedimentation, rinsed with distilled water, and dried. The handle is disinfected using disinfection wipes (Ebiox Ltd., Cheshire, UK).

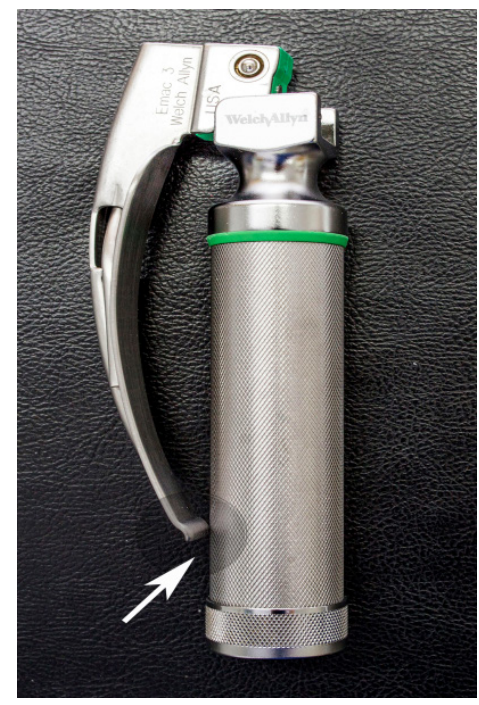

Figure 1. Photograph showing that the tip of the blade contacts an area on the knurled surface of the handle (arrow) when in the off position.

Blades are usually packed and sealed in synthetic resin packing materials or left unpacked with the handles. In this case, the instrument would be contaminated by the cart itself, other equipment, medical team members' hands, or a droplet or airborne microbe even if cleaning and disinfection is completed $[14,15]$. Based on recent studies, the handles and blades of ready-for-patient-use laryngoscopes will have bacteria that can cause a nosocomial infection $[5,6,9,12,13,16,17]$.

There have been studies about laryngoscope equipped in the operation room, but the contamination rate of laryngoscopes in other environments, such as entire wards including the emergency room, has not been evaluated. The contamination rate refers to the positive ratio or proportion of bacterial cultivation from target sample collections. Some hospitals, including our study's target hospitals, use disinfection wipes and follow a high-level disinfection protocol that destroys some spore-forming bacteria and most microorganisms on the laryngoscope handle [18]. However, no study has assessed the contamination rate after disinfection.

Therefore, the primary goal of this study was to evaluate the contamination rate of laryngoscope blades and handles stored in emergency crash carts of departments, excluding the operation room. The secondary goal was to determine the distribution of microorganisms of the laryngoscope. Moreover, we compared the contamination rates of two medical institutions that use the same disinfection method, and we assessed the contamination rates according to the frequency of laryngo- 
scope use to determine the factors that affect bacterial contamination of laryngoscopes.

\section{METHODS}

In September 2014, this prospective, descriptive study was conducted in wards, including emergency rooms, of two tertiary teaching hospitals located in Gyeonggi and Chungcheong Province (hospitals 1 and 2). Laryngoscopes identified as ready for patient use were included, and laryngoscopes that were not disinfected were excluded from the study. Excluding the operating room, 162 laryngoscope blades and 79 handles were collected; 148 laryngoscope blades and 71 handles were selected to acquire specimens for bacterial cultivation. The handle top and handle's knurled surface were regarded as separate samples.

One researcher collected all the samples and wore new, sterilized gloves for each sample to minimize contamination. The parts of laryngoscopes for sample collection (Figure 2) included the (A) inside part of the blade, (B) front part of the handle top, and (C) front part of the handle's knurled surface. Using sterile swabs (Stuart Agar Gel Transport Swabs; Copan Italia SpA, Brescia, Italy), the laryngoscope's target parts were wiped five times, and then the parts were rolled and placed in a transport medium; the samples were transported to the laboratory the same day. Two hours after sample collection, the samples were inoculated in glucose cystine blood agar (BAP;

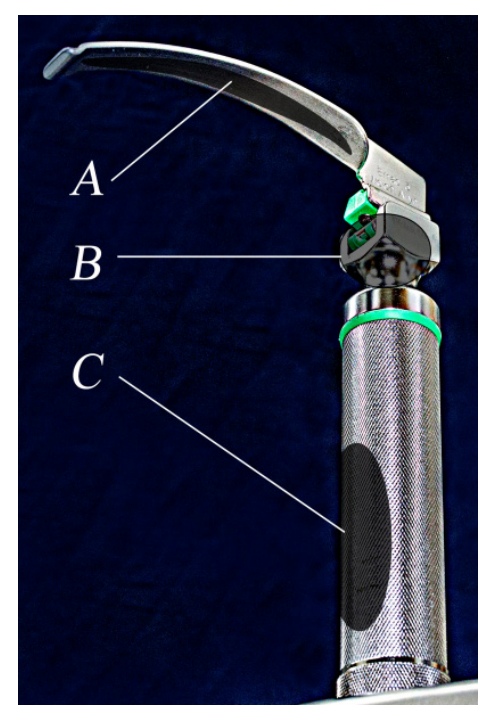

Figure 2. Photograph showing the three sampling sites (AC) on each of the ready-for-patient-use laryngoscopes (148 handles and 71 blades).
Hanil Komed Co., Anyang, Korea) and incubated aerobically at $35^{\circ} \mathrm{C}$. After 18 hours of incubation, the colonies of microorganisms were counted and identified using standard microbiological methods using the Vitek-2 System (BioMérieux, Lyon, France). A sample was considered positive if there was any microbial growth on the BAP.

To compare bacterial contamination rates depending on direct laryngoscopic orotracheal intubation (DLOTI) frequencies, we selected a critical care area (CCA) for departments with more than 10 times of DLOTI frequencies per year and a noncritical care area (NCCA) for departments with less than 10 times of DLOTI frequencies per year. The CCAs included emergency rooms, intensive care units (ICUs), and neonatal ICUs, whereas NCCAs included outpatient and general wards and all types of examination rooms. To compare bacterial contamination rates of each hospital, laryngoscopes at two hospitals were selected as a separate target specimen.

The number of DLOTIs for each department of the two hospitals was collected from the electronic medical record system. After specimen collection, a unique number was recorded on transport swabs, and the collected part and unique number were recorded on a survey form. The data collection period was three days.

Collected data were analyzed using SPSS version 18.0 (SPSS Inc., Chicago, IL, USA). Categorical variables, such as the type of microorganism and degree of contamination, are presented as frequencies and percentages. Comparative analyses of the degree of contamination by the parts of the laryngoscope, DLOTI frequency, and hospital were performed using the $\chi^{2}$ test and Fisher exact test; a $p<0.05$ was considered significant.

The current study was approved by the institutional review board (IRB) of our medical institution (IRB approval no. NON 2014-002).

\section{RESULTS}

\section{Bacterial Contamination Rate of Laryngoscopes}

Two hundred ninety samples were obtained over the 2-day sampling period (blade, 148; handle top, 71 ; handle's knurled surface, 71$)$. The contamination rates were $18.2 \%$ (27/148) for blades, 5.6\% (4/71) for handle tops, and 28.2\% (20/71) for handles' knurled surfaces (Table 1). Compared with handle tops, the contamination rate of the handles' knurled surfaces was significantly higher $(p<0.001)$. The blades and handles of the laryngoscopes were classified in different groups accord- 
Table 1. Stratification of contamination by the sampling variables

\begin{tabular}{|c|c|c|c|c|c|c|c|c|c|}
\hline & \multicolumn{2}{|c|}{ Blade } & & \multicolumn{2}{|c|}{ Handle top } & & \multicolumn{2}{|c|}{ Handle knurled surface } & \\
\hline & n (\%) & $p$-value & & n (\%) & $p$-value & & n (\%) & $p$-value & \\
\hline Area $^{1}$ & & 0.52 & & & 0.60 & & & 0.003 & \\
\hline CCA & 15/74 (20.3) & & A: 6 & 2/24 (8.3) & & A: 2 & $12 / 24(50.0)$ & & A: 6 \\
\hline & & & B: 9 & & & B: 0 & & & B: 6 \\
\hline NCCA & $12 / 74(16.2)$ & & A: 7 & $2 / 47(4.3)$ & & A: 1 & 8/47 (17.0) & & $A: 7$ \\
\hline & & & B: 5 & & & B: 1 & & & B: 1 \\
\hline Hospital & & 0.60 & & & 0.31 & & & 0.02 & \\
\hline A & 13/78 (16.7) & & CCA: 6 & $3 / 31(9.7)$ & & CCA: 2 & 13/31 (41.9) & & CCA: 6 \\
\hline & & & NCCA: 7 & & & NCCA: 1 & & & NCCA: 7 \\
\hline B & $14 / 70(20.0)$ & & CCA: 9 & $1 / 40(2.5)$ & & CCA: 0 & $7 / 40(17.5)$ & & CCA: 6 \\
\hline & & & NCCA: 5 & & & NCCA: 1 & & & NCCA: 1 \\
\hline Total & $27 / 148(18.2)$ & & & $4 / 71(5.6)$ & & & 20/71 (28.2) & & \\
\hline
\end{tabular}

CCA, critical care area; NCCA, non-critical care area.

${ }^{1}$ Two areas defined according to the frequency of intubation attempts.

ing to the level of disinfection depending on the instrument as well as the disinfection instructions followed. Therefore, an equivalence check was not performed.

\section{Microorganisms Isolated}

We isolated 17 genera, 22 species from 64 isolated strains. Bacillus spp. were most frequent (12 cases, 18.8\%), followed by coagulase-negative staphylococci and Acinetobacter spp. (10 cases each, 15.6\%), Micrococcus spp. (9 cases, 14.1\%), and other microorganisms, such as Kocuria rosea, viridans streptococci, Candida spp., Corynebacterium spp., etc. (28 cases each on laryngoscope blades, 5 cases each on handle top, and 31 cases each on handles' knurled surfaces (Table 2). In the case of more than 2 isolated strains, there were 4 cases on laryngoscope blades, 1 case on a handle top, and 7 cases on handles' knurled surfaces.

\section{Contamination Rate Depending on the Direct Laryngoscopic Orotracheal Intubation Frequency and Hospitals}

Laryngoscopes were collected as target samples from 1 emergency room, 2 ICUs, 1 neonatal ICUs, 26 wards and outpatient clinics, and 8 examination-related departments of hospital 1, a tertiary teaching hospital with 960 beds. Laryngoscopes were collected as target samples from 1 emergency room, 3 ICUs, 1 neonatal ICU, 25 wards and outpatient clinics, and 7 examination-related departments of hospital 2, a tertiary teaching hospital with 890 beds.

At these 2 hospitals, CCAs had 7119 DLOTI attempts and NC-
Table 2. Types of microorganisms isolated

\begin{tabular}{|c|c|c|c|c|}
\hline \multirow[b]{2}{*}{ Microorganisms cultured } & \multicolumn{4}{|c|}{ Samples with positive growth } \\
\hline & $\begin{array}{l}\text { Total } \\
\text { n (\%) }\end{array}$ & Blade & $\begin{array}{l}\text { Handle } \\
\text { top }\end{array}$ & $\begin{array}{l}\text { Handle } \\
\text { knurled } \\
\text { surface }\end{array}$ \\
\hline Bacillus spp. & $12(18.8)$ & 2 & 1 & 9 \\
\hline Coagulase-negative staphylococci & $10(15.6)$ & 7 & 1 & 2 \\
\hline Staphylococcus aureus & $2(3.1)$ & 2 & 0 & 0 \\
\hline Acinetobacter spp. & $10(15.6)$ & 2 & 2 & 6 \\
\hline Micrococcus spp. & $9(14.1)$ & 6 & 1 & 2 \\
\hline Kocuria rosea & $3(4.7)$ & 2 & 0 & 1 \\
\hline Viridans streptococci & $3(4.7)$ & 1 & 0 & 2 \\
\hline Candida spp. & $2(3.1)$ & 0 & 0 & 2 \\
\hline Corynebacterium spp. & $2(3.1)$ & 0 & 0 & 2 \\
\hline Aerococcus viridans & $1(1.6)$ & 1 & 0 & 0 \\
\hline Brevundimonas vesiculari & $1(1.6)$ & 0 & 0 & 1 \\
\hline Moraxella spp. & $1(1.6)$ & 1 & 0 & 0 \\
\hline Ochrobactrum anthropi & $1(1.6)$ & 0 & 0 & 1 \\
\hline Pantoea spp. & $1(1.6)$ & 0 & 0 & 1 \\
\hline Paracoccus yeei & $1(1.6)$ & 0 & 0 & 1 \\
\hline Stenotrophomonas maltophilia & $1(1.6)$ & 1 & 0 & 0 \\
\hline Pseudomonas spp. & $1(1.6)$ & 1 & 0 & 0 \\
\hline Kytococcus sedentarius & $1(1.6)$ & 1 & 0 & 0 \\
\hline Unidentified mold & $2(3.1)$ & 1 & 0 & 1 \\
\hline Total & $64(100)$ & 28 & 5 & 31 \\
\hline
\end{tabular}

CAs had 173 DLOTI attempts over 3 years (Table 3). In the case of laryngoscope blades, the contamination rates were $20.3 \%$ (15/74) in CCAs and 16.2\% (12/74) in NCCAs without a significant difference between these 2 groups. In the case of handle tops, the contamination rates were $8.3 \%(2 / 24)$ in CCAs and 
Table 3. Total number of intubation attempts over the past 3 years

\begin{tabular}{cc}
\hline & Intubations attempts \\
\hline Area ${ }^{1}$ & 7119 \\
CCA & 173 \\
NCCA & \\
Hospital & 3486 \\
A & 3806 \\
B & 7292 \\
Total
\end{tabular}

CCA, critical care area; NCCA, non-critical care area.

${ }^{1}$ Two areas defined according to the frequency of intubation attempts.

4.3\% (2/47) in NCCAs without a significant difference between these 2 groups. In contrast, for handles' knurled surfaces, the contamination rates were $50.0 \%(12 / 24)$ in CCAs and $17.0 \%$ (8/47) in NCCAs with a significantly higher contamination rate in CCAs, indicating that the contamination rate of the handles' knurled surfaces was influenced by its frequency of use ( $p=$ 0.003) (Table 1).

Hospital 1 had 3486 DLOTI attempts and hospital 2 had 3806 DLOTI attempts over 3 years (Table 3). In the case of laryngoscope blades, the contamination rates were $16.7 \%$ $(13 / 78)$ at hospital 1 and $20.0 \%$ (14/70) at hospital 2 without a significant difference between these 2 groups. In the case of handle tops, the contamination rates were $9.7 \%(3 / 31)$ at hospital 1 and 2.5\% (1/40) at hospital 2 without a significant difference between these 2 groups. However, for handles' knurled surfaces, the contamination rates were $41.9 \%$ (13/31) at hospital 1 and $17.5 \%$ (7/40) at hospital 2 with a significantly higher contamination rate for hospital 1, indicating that the contamination rate of handles' knurled surfaces was different at each medical institution $(p<0.05)$ (Table 1).

\section{DISCUSSION}

The contamination rates from previous studies since 2008 $[12,16,17,19-21]$, when the US CDC suggested disinfection instructions, were 42.0 to $57.3 \%$ of laryngoscope blades and 69.2 to $86.0 \%$ of handles. These values were more than those of the present study were, and this result may be caused by differences in each institution's disinfection instructions, specimen collection method, sample delivery, culture method, and type of department that used a laryngoscope. The present study has several strengths: the contamination rate was assessed in terms of factors such as the DLOTI frequency and different in- stitutions; sample size was large; environments other than the operating room were assessed; and we found that the disinfection status of reused laryngoscopes was still unacceptable, indicating this as a risk factor for nosocomial infection.

In the case of laryngoscope blades, the contamination rate of each department did not differ depending on the medical institution and DLOTI frequencies. The reason for this finding is that they are designated and relatively specific disinfection instructions for laryngoscope blades compared to handles. Both institutions and all the departments used the same disinfectants from sedimentation for more than 10 minutes, rinsed with distilled water, dried with sterilized gauze or linen, and maintained the disinfected packing material properly to ensure high-level disinfection [8]. The status of sealing, however, was not indicated, which caused unsealed packing material as the source of contact, droplet, or airborne contamination $[14,15]$. Practically, it is difficult to ensure that the packages are sealed in an aseptic condition after disinfection using the target hospitals' current disinfection instructions for laryngoscopes, as there are shortages of nurses; thus, alternatives that are more effective and not necessarily cost effective are needed.

Regarding handles, samples were collected from 2 parts, and the contamination rate was significantly higher for handles' knurled surfaces than for the handle top. This result represents the effectiveness of disinfecting a smooth surface with wipes. Moreover, the handles' knurled surfaces had the highest contamination rate, which was significantly different according to the DLOTI frequency and institution. This result is considered to not be mainly caused by specific disinfection instructions. In other words, it is possible to have a qualitative difference according to each person in charge of disinfecting the laryngoscope by the guideline, such as just wiping it with a disinfection wipe. Structural problems such as the grid-type knurled surface cause contamination [12,13], but differences according to the intubation frequency and medical institution will be regarded as crucial reason for contamination; thus, the reinforcement and standardization of disinfection instructions are required. Additionally, unclear regulations for maintenance are another cause of bacterial contamination that require improvement.

Bacillus spp., coagulase-negative staphylococci, Acinetobacter spp., Micrococcus spp., and Corynebacterium spp., the highest isolated strain (67.2\%), can spread through the air [22]. To prevent bacterial contamination, it is mandatory to seal the laryngoscope following high-level disinfection. There were 10 cases of coagulase-negative staphylococci (15.6\%) and 2 cases 
of Staphylococcus aureus (3.1\%) cultivated with common skin flora and Micrococcus spp. This result implies that the manager's hand caused contamination after disinfection; thus, hand hygiene and the use of gloves needs enforced to prevent this type of contamination. Viridans streptococci and Corynebacterium spp. are oral cavity flora; thus, these results of cultures, mainly from the handles with a knurled surface, imply the following: 1) during DLOTI, the blade is contaminated by the oral cavity; and 2) when the blade is folded, the handle's knurled surface comes into contact with the contaminated blade. To prevent this contamination route, the structure of the laryngoscope should be improved so it is not necessary to touch the handle surface even when laryngoscope blades are folded. Pathogenic microorganisms such as Acinetobacter spp. and Candida spp. were isolated at high rates for various strains, including Kocuria rosea, Aerococcus viridans, Brevundimonas vesicularis, Ochrobactrum anthropi, Pantoea spp., Paracoccus yeei, Stenotrophomonas maltophilia, and Kytococcus sedentarius, making an opportunistic infection possible.

In the current study, a single-culture method was used; therefore, it was not possible to determine antibiotic resistant isolates, anaerobes, viruses, and prions. The approved microorganism contamination (i.e., the approved limit portion of microorganism contamination) was limited; however, our findings implied the possibility of infection by other pathogenic microorganisms on the laryngoscope. Future studies should assess more microorganisms, and the clinical result of DLOTI should be followed for a longer period.

According to our study's results, the frequency of use and medical institution were factors affecting laryngoscope contamination with complex effects on the maintenance status, hand hygiene status of the person in charge, and laryngoscope structure. Additionally, current laryngoscope disinfection instructions do not suggest sterilization, especially for handles, and a relatively low-level disinfection is recommended for blades without specific methods [7-9]. Therefore, the risk of nosocomial infection through laryngoscopes is prevalent. Moreover, there are limitations on high-level disinfection due to the concerns about failure of some subsidiary parts, such as the handle's battery, wires, and light bulb. To overcome this issue, it has been suggested that a sheath or single-use laryngoscope blades should be used for airway intubation $[10,23]$. In the case of a sheath, however, it can be damaged during use and contaminated during the covering and peeling off process [10]. Single-use laryngoscope blades can also be contaminated during the folding process, and if they are made of a plastic material, the blades bend and obstruct the physician's view [24]. For single-use, plastic laryngoscopes, which have been recently introduced in the clinical field, have blades and handles made of a hard material that are integrated as a single unit; therefore, it is not possible to fold or bend them. These should be approved considering their effectiveness in terms of the intubation success rate, efficiency, and cost.

In conclusion, the laryngoscope is still a risk factor of nosocomial infection, and the different contamination rates from handles' knurled surfaces depending on the medical institution and DLOTI frequency implies the possibility of a qualitative difference caused by current handle disinfection method, which is affected by various factors. Based on our study's results, we suggest (1) developing more strict, high-level, standardized disinfection instructions and maintenance instructions, (2) improving the structure of laryngoscopes so they are more practical and efficient, and (3) considering the use of single-use, one-piece laryngoscopes with blades that are not folded.

\section{ACKNOWLEDGEMENTS}

This work was supported by the Soonchunhyang University Research Fund.

\section{CONFLICT OF INTEREST}

The authors have no conflicts of interest associated with the material presented in this paper.

\section{ORCID}

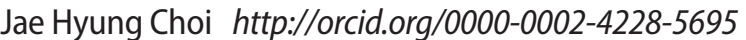

Young Soon Cho http://orcid.org/0000-0002-6843-9517

\section{REFERENCES}

1. Hall JR. Blood contamination of anesthesia equipment and monitoring equipment. Anesth Analg 1994;78(6):1136-1139.

2. Morell RC, Ririe D, James RL, Crews DA, Huffstetler K. A survey of laryngoscope contamination at a university and a community hospital. Anesthesiology 1994;80(4):960.

3. Lee SO. A survey on hospital infection. J Nurs Acad Soc 1993; 23(3):325-338 (Korean).

4. Kim JM. Current state of nosocomial infections in Korea. Kore- 
an J Med 1999;57(4):572-577 (Korean).

5. Cullen MM, Trail A, Robinson M, Keaney M, Chadwick PR. Serratia marcescens outbreak in a neonatal intensive care unit prompting review of decontamination of laryngoscopes. J Hosp Infect 2005;59(1):68-70.

6. Jones BL, Gorman LJ, Simpson J, Curran ET, McNamee S, Lucas $C$, et al. An outbreak of Serratia marcescens in two neonatal intensive care units. J Hosp Infect 2000;46(4):314-319.

7. Rutala WA, Weber DJ; Healthcare Infection Control Practices Advisory Committee (HICPAC). Guideline for disinfection and sterilization in healthcare facilities, 2008 [cited 2017 Apr 28]. Available from: https://www.cdc.gov/hai/pdfs/disinfection_ nov_2008.pdf.

8. Ministry of Government Legislation. Guidelines for medical instruments and material disinfection in healthcare facilities [cited 2014 Aug 13]. Available from: http://www.law.go.kr/admRulLsInfoP.do?admRulSeq =2000000014476 (Korean).

9. AORN Recommended Practices Committee. Recommended practices for cleaning, handling and processing anesthesia equipment. AORN J 2005;81(4):856-857, 860-870.

10. Muscarella LF. Reassessment of the risk of healthcare-acquired infection during rigid laryngoscopy. J Hosp Infect 2008;68(2): 101-107.

11. Kim HY, Lee EJ, Jang EK, Park YA. A study for infection control standards for medical devices in NICU. J Korean Clin Nurs Res 2010;16(2):69-84 (Korean).

12. Williams D, Dingley J, Jones C, Berry N. Contamination of laryngoscope handles. J Hosp Infect 2010;74(2):123-128.

13. Negri de Sousa AC, Levy CE, Freitas MI. Laryngoscope blades and handles as sources of cross-infection: an integrative review. J Hosp Infect 2013;83(4):269-275.

14. Esler MD, Baines LC, Wilkinson DJ, Langford RM. Decontamination of laryngoscopes: a survey of national practice. Anaesthesia 1999;54(6):587-592.
15. Simmons SA. Laryngoscope handles: a potential for infection. AANA J 2000;68(3):233-236.

16. Lowman W, Venter L, Scribante J. Bacterial contamination of re-usable laryngoscope blades during the course of daily anaesthetic practice. S Afr Med J 2013;103(6):386-389.

17. Call TR, Auerbach FJ, Riddell SW, Kiska DL, Thongrod SC, Tham SW, et al. Nosocomial contamination of laryngoscope handles: challenging current guidelines. Anesth Analg 2009;109 (2):479-483.

18. EBIOX. Trionic: advanced environmental hygiene [cited 2014 Mar 2]. Available from: http://www.ebiox.co.uk/products/ view/trionic/.

19. Qureshi T, Barbut F, Pernet P, Neyme D, Maury E, Offenstadt G. Laryngoscope handles in a medical intensive care unit: the level of bacterial and occult blood contamination. J Hosp Infect 2008;68(1):94-95.

20. Telang R, Patil V, Ranganathan P, Kelkar R. Decontamination of laryngoscope blades: is our practice adequate? J Postgrad Med 2010;56(4):257-261.

21. Howell V, Thoppil A, Young H, Sharma S, Blunt M, Young P. Chlorhexidine to maintain cleanliness of laryngoscope handles: an audit and laboratory study. Eur J Anaesthesiol 2013; 30(5):216-221.

22. Hwang SH, Park DU, Ha KC, Cho HW, Yoon CS. Airborne bacteria concentrations and related factors at university laboratories, hospital diagnostic laboratories and a biowaste site. J Clin Pathol 2011;64(3):261-264.

23. Machan MD, Monaghan WP, McDonough J, Hogan G. Emerging evidence in infection control: effecting change regarding use of disposable laryngoscope blades. AANA J 2013;81(2): 103-108.

24. Anderson K, Gambhir S, Glavin R, Kinsella J. The use of an anaesthetic simulator to assess single-use laryngoscopy equipment. Int J Qual Health Care 2006;18(1):17-22. 